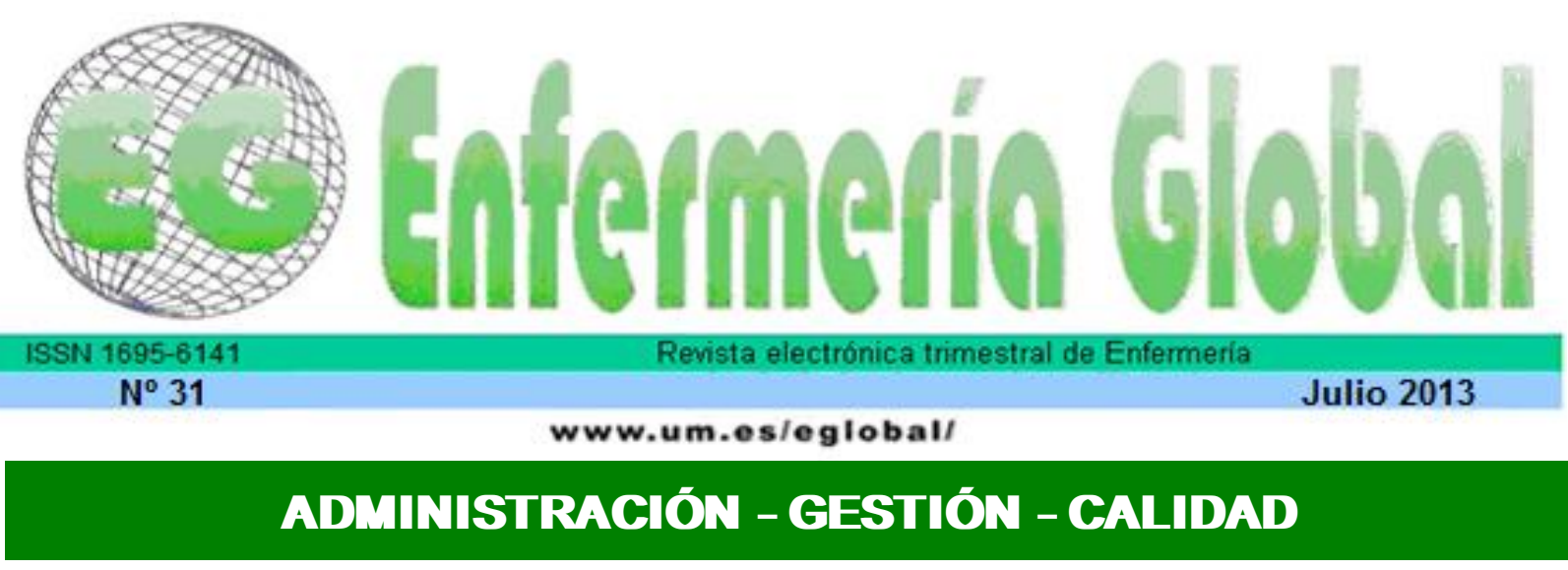

\title{
Búsqueda de información sobre salud a través de internet
}

Searching for information on health through internet

\begin{abstract}
*Villaescusa Martínez, Víctor **Sáez Villar, Lorena
*Enfermero. Servicio Riojano de Salud. E-mail: vivillaescum@hotmail.com **Técnico en Cuidados
\end{abstract}

Auxiliares de Enfermería. Hospital Viamed Los Manzanos. La Rioja. España.

Palabras clave: Internet; búsqueda; Información; Salud.

Keywords: Internet; Research; Information; Health

\section{RESUMEN}

Internet es una herramienta en constante crecimiento y tremendamente influyente en la población. Nos proporciona información prácticamente ilimitada, pero no toda es confiable.

Objetivo: Conocer si los pacientes hacen uso de Internet para informarse sobre salud, si lo hacen previa o posteriormente a consultar con profesionales sanitarios y credibilidad que le dan a la información recibida. Así mismo, observar la evolución de las búsquedas a lo largo del tiempo y la influencia generacional su uso.

Material y método: Estudio descriptivo transversal mediante un cuestionario autoadministrado a 150 pacientes hospitalizados y de consultas, compuesto por cuatro ítems sobre usos habituales de búsqueda en Internet relacionados con la salud. Posteriormente se estudian comparativamente los resultados obtenidos respecto a datos del INE (Instituto Nacional de Estadística) correspondientes a años anteriores.

Resultados: $65 \%$ de los pacientes usan Internet para informarse sobre salud. Entre los pacientes menores de 60 años lo hacen el $85.5 \%$ mientras que entre los mayores de 60 el $24.5 \%$. Un $47.4 \%$ se informa antes y otro $47.4 \%$ lo hace después de contactar con profesionales (5.2\% antes y después). La credibilidad media es de 5.67 puntos sobre 10. Las búsquedas en Internet sobre salud siguen aumentando con el paso de los años.

Conclusiones: Internet es una herramienta de uso habitual para informarse sobre salud, sobre todo entre los más jóvenes, pero la credibilidad otorgada a esa información no es muy alta, por lo que es necesario un mayor control de la información existente, así como una educación sanitaria correcta, adecuada y continuada por parte de los profesionales.

\section{ABSTRACT}

Internet is a tool in constant growth and has a tremendous influence on the population. Although it provides almost unlimited information, not all this information is reliable. 
Goal: to get to know if patients make use of the Internet to find out about health; if they do it before or after consulting health professionals and the credibility they give to the obtained information. Furthermore, notice the progress of the searches over time and the generational influence on the use of this tool.

Methodology: descriptive and cross-sectional research through a questionnaire to 150 in-patients and out-patients with four health-related items about typical uses of the Internet. Subsequently, the research results were compared with data from the NSI (National Statistical Institute) in previous years.

Results: $65 \%$ of the patients make use of the Internet to learn about health. Among patients under 60 years do $85.5 \%$ in front of patients above $60,24.5 \%$. A $47.4 \%$ reported earlier and another $47.4 \%$ do after contact with professionals $(5.2 \%$ before and after). The credibility average they give to the information is 5.67 points on 10 . Internet searches about health continue to grow with the passage of the years.

Conclusions: Internet is a tool commonly used by patients to learn about health, especially among the young, but the credibility given to this information is not very high, so it is necessary a greater control of the existent information, as well as a proper health education, adequate and continued by professionals.

\section{INTRODUCCIÓN}

Desde su origen en la década de los 60, Internet ha tenido una evolución meteórica. En apenas 25 años, la idea de Robert Taylor de interconectar ordenadores para que los investigadores que estuvieran haciendo un trabajo similar en diferentes lugares del país pudieran compartir recursos y resultados más fácilmente, ha pasado a ser un instrumento prácticamente universal y de uso diario para la búsqueda de información (1), (2)

Sin embargo, el hecho de disponer de tanta información de manera prácticamente inmediata, también conlleva ciertos peligros, sobre todo en lo que a la veracidad de la misma se refiere ${ }^{(3)}$.

Dentro del ámbito sanitario, esta evolución también se ha hecho patente, y con el paso de los años se observa un cambio hacia una sociedad cada vez mas informada sobre salud en Internet, hasta el punto de adoptar nuevos términos para nuestro vocabulario como el de "E-salud", para aludir a la práctica de cuidados sanitarios apoyada en las Tecnologías de la Información y las Comunicaciones (TIC) ${ }^{(4-6)}$.

Así mismo, al incremento de la información que aparece en la red hay que sumar el crecimiento de las búsquedas relacionadas con la salud por parte de los usuarios, que cada vez son más elevadas. De hecho, y según los datos del Instituto Nacional de Estadística (INE), en España se estima que del total de usuarios de Internet mayores de 16 años, en el año 2003 habían buscado temas relacionados con la salud el 19,5\% de ellos, en 2007 el $41,1 \%$ y las cifras de 2011 lo sitúan ya en un $56,9 \%{ }^{(7)}$.

Internet es a su vez tremendamente influyente en la adopción de estilos de vida saludables o no saludables ${ }^{(8),(9)}$, siendo el estilo de vida el determinante de salud más condicionante de la misma ${ }^{(10)}$.

Por otro lado, también se constata que es un medio de comunicación muy difícil de controlar y con deficiencias, ya que a pesar de haberse convertido en el instrumento fundamental de la "transmisión de conocimientos en salud", la información no pasa por filtros editoriales que puedan asegurar la veracidad de la misma ${ }^{(11)}$. De ahí que la confiabilidad que podemos tener de Internet no es del $100 \%{ }^{(12-14)}$. 
En cuanto a la taxonomía de intervenciones enfermeras, dentro del nivel 3 (Intervenciones), y en el tercer campo (Conductual, cuidados que apoyan el funcionamiento psicosocial y facilitan los cambios de estilo de vida), y en concreto en la clase " $S$ " (educación de los pacientes), se encuentra la educación sanitaria (actividad 5510) ${ }^{(15)}$.

Es por tanto función de la enfermería, determinar el conocimiento sanitario existente, considerar la accesibilidad y las preferencias del consumidor y ayudar a clarificar las creencias y valores sanitarios. En definitiva, proporcionar una educación sanitaria adecuada.

De este modo y puesto que se trata de una herramienta tan influyente en los conocimientos de la población con respecto a la salud y en la adopción de estilos de vida saludables o no saludables y dado que no es completamente confiable, es de gran importancia para los profesionales sanitarios saber cuáles son los usos de Internet por parte de nuestros pacientes, así como la credibilidad que le otorgan a la información obtenida. Por ello nos marcamos como objetivo saber cuántos de nuestros pacientes se informan sobre salud a través de la red, si lo hacen antes o después de contactar con profesionales sanitarios y si consideran veraz la información obtenida. Además queremos conocer la influencia que tiene la edad en el uso de Internet en nuestra sociedad y si continúa la evolución ascendente en cuanto a las búsquedas relacionadas con la salud.

\section{MATERIAL Y MÉTODOS}

\section{Tipo de diseño}

Estudio descriptivo de corte transversal.

\section{Muestra}

La muestra para el estudio resulta un muestreo consecutivo. La recogida de la información se realizó en el Hospital Viamed Los Manzanos.

Consideramos que los pacientes que acuden al Hospital Viamed Los Manzanos contienen en su composición una representatividad sociocultural de la población objeto. A partir de aquí se aplicó un cuestionario a los pacientes ingresados y de consultas a lo largo del 21 y 22 de Junio de 2012 hasta obtener un total de 150 cuestionarios.

El único criterio imprescindible para la participación fue el dominio del castellano para comprender y cumplimentar el cuestionario.

\section{Instrumentos de evaluación}

\section{- Desarrollo y características}

Para la elaboración del cuestionario se partió de la basede que para diseñar y aplicar un cuestionario debemos tener un balance adecuado entre la sencillez y la claridad pero sin caer en lo superficial ${ }^{(16)}$. 
Atendiendo a estos propósitos se eligió un cuestionario con preguntas dicotómicas para conocer los usos de Internet por parte de los pacientes y para valorar la credibilidad de la información obtenida, la escala tipo Likert, al tratarse esta el sistema más sencillo y más utilizado de construcción de escalas y sus características psicométricas no son al menos inferiores (o incluso pueden considerarse superiores) a las de otros tipos de escalas ${ }^{(17)}$. En este caso contemplamos la creación de un ítem diseñado a tal efecto, tomando como referencia para la elaboración del mismo las cuestiones similares en cuanto a contenido utilizadas por el Centro de Investigaciones Sociológicas en el Barómetro Sanitario 2011 para sus preguntas ponderativas ${ }^{(18)}$.

De este modo, se formularon tres ítems con preguntas dicotómicas y una tipo Likert, de tal manera que el cuestionario no se prolongase excesivamente en el tiempo para evitar el cansancio o rechazo por parte del encuestado dado que se previó la posibilidad de que en algún caso, especialmente entre los pacientes hospitalizados, la cumplimentación de un cuestionario no fuese de su agrado o pudiese ocasionar un esfuerzo. Además se adaptó el lenguaje de los ítems adecuándolo a personal no sanitario para obtener una buena comprensión por parte de los mismos.

La redacción de los ítems citados evitó sesgos cognitivos que pudieran inducir juicios de valor por parte del encuestado y dar éste respuestas socialmente aceptables ${ }^{(19)}$.

El coeficiente de consistencia interna del cuestionario para las preguntas dicotómicas fue de 0.77 utilizando la fórmula Kuder Richardson ${ }^{(20)}$ ( $K R=0.77$ ). La pregunta ponderativa tipo Likert se obtuvo a partir del Barómetro Sanitario 2011, utilizando un enunciado similar.

Así pues, se estructura metodológicamente el cuestionario ajustándose a los objetivos que se pretenden y valorando de forma afirmativa la adecuación a la muestra.

\section{- Aplicación}

Recogida de la información a través de un cuestionario autoadministrado de carácter voluntario y anónimo. Se entregó a cada paciente un cuestionario compuesto por 4 ítems, además de un apartado para cumplimentar con la edad del mismo. Los tres primeros ítems fueron preguntas cerradas, dicotómicas, en las que se pretendía obtener información sobre:

1.- Si el encuestado había hecho uso de Internet para buscar información sobre temas relacionados con la salud en alguna ocasión.

2.- Si se había informado en Internet sobre su proceso actual.

3.- Si buscó la información antes o después de consultar con profesionales sanitarios.

El último ítem contaba con una pregunta ponderativa para evaluar la calidad de la información obtenida en Internet, en concreto una escala de valoración Likert 1-10, siendo 10 la máxima veracidad y 1 la mínima.

\section{Análisis estadístico}

Para el análisis de los datos se utilizó un software perteneciente al paquete ofimático Microsoft Office en concreto Microsoft Excel usando una hoja de cálculo diseñada ex professo en la que se aplicaron medidas de tendencia central (media, moda y mediana) y proporciones, primero de forma general a toda la muestra y luego 
dividiéndola en dos grupos (mayores y menores de 60 años). Para el proceso de validación del cuestionario se transportaron los datos a una hoja de cálculo de confiabilidad diseñada para excel por Del Siegle. (Reliability calculador $\left.\AA^{\circledR}\right)$.

Se compararon los datos obtenidos en la encuesta con los ya existentes en el INE de los años 2003, 2007 y 2011.

\section{RESULTADOS}

Del total de los cuestionarios obtenidos el $44.7 \%$ se realizó a pacientes hospitalizados en dos plantas de patología mixta (médica y/o quirúrgica), en diversos estados de evolución. El restante $55.3 \%$ se realizó a pacientes que acudieron a consultas externas de diversas especialidades, tales como Traumatología, Oftalmología, Cirugía, etc.

Se obtuvieron un total de 150 cuestionarios. 4 de los mismos fueron declarados nulos por respuestas incongruentes. 3 de ellos fueron obtenidos de pacientes menores de 16 años, por lo que estarán incluidos en las estadísticas generales del estudio, pero serán eliminados al compararlos con los datos del INE, que utiliza datos obtenidos en individuos mayores de 16 años.

La edad media de los individuos encuestados fue de 51.6 años, siendo 9 años la edad del más joven y 91 años la del más mayor. El 58\% eran mujeres frente al $42 \%$ de hombres.

Del total de cuestionarios validados (146), el $65.07 \%$ habían hecho uso de Internet para buscar información sobre temas referentes a la salud en alguna ocasión, mientras que el $34.93 \%$ no lo habían hecho en ninguna ocasión. (Figura 1).

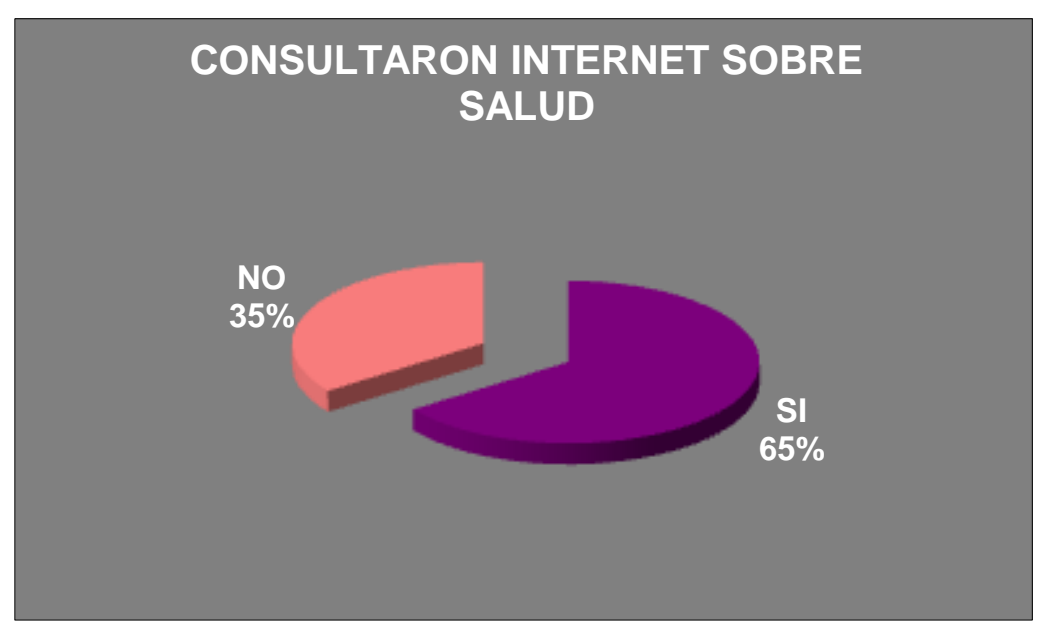

Figura 1: Porcentaje de pacientes que han consultado Internet para informarse sobre salud en alguna ocasión.

Dividiendo la muestra en dos grupos de edad, menores y mayores de 60 años: del total de pacientes menores de 60 años, el $85.5 \%$ sí que hizo uso de la red para informarse sobre temas de salud, mientras que el $14.5 \%$ no lo hizo. De los pacientes mayores de 60 años, el $24.5 \%$ se informó en la red sobre temas de salud, mientras que el $75.5 \%$ no lo había hecho nunca. (Figura 2). 


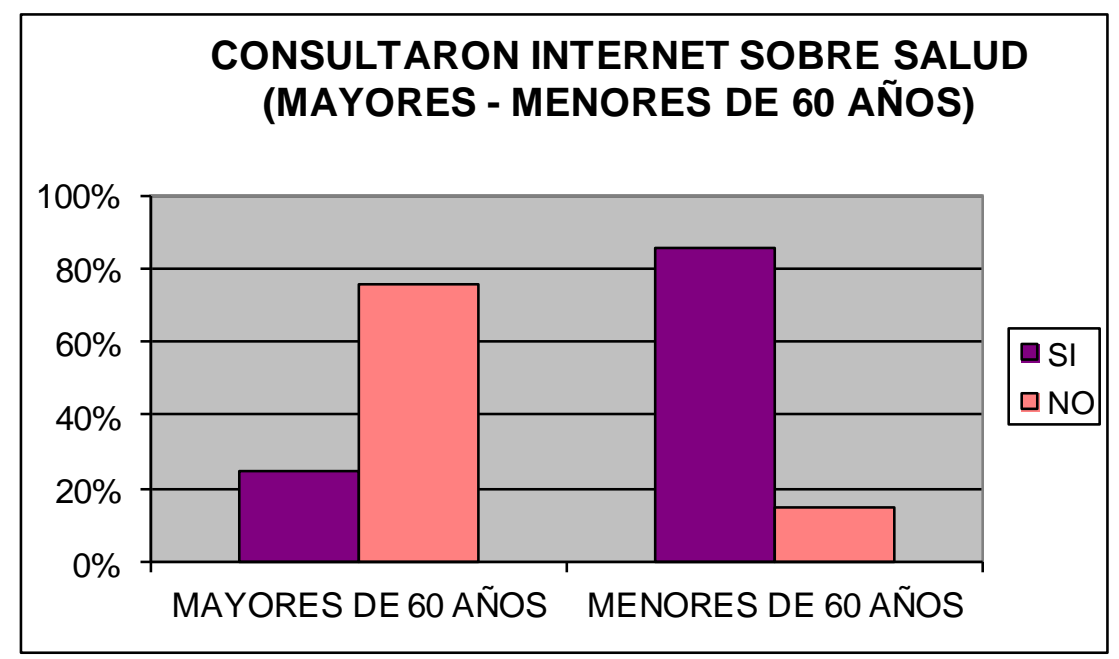

Figura 2: Porcentaje de pacientes que han consultado Internet para informarse sobre salud en alguna ocasión. Comparación mayores de 60 años/menores de 60 años.

Del total de personas que en alguna ocasión habían obtenido información de la red en temas referentes a la salud, el $71.58 \%$ ya se había informado para su proceso actual, mientras que el $28.42 \%$ no lo había hecho.

Del total de encuestados que obtienen información en la red sobre temas de salud el $47.4 \%$ consulta Internet antes de ponerse en contacto con su médico, otro $47.4 \%$ lo hace después y un $5.2 \%$ lo hace antes y después.

La credibilidad media que se le dio a la información obtenida de Internet fue de 5.67 sobre un máximo de 10, no apreciándose diferencias significativas por grupos de edad. La moda fue 5 y la mediana 6.(Figura 3).

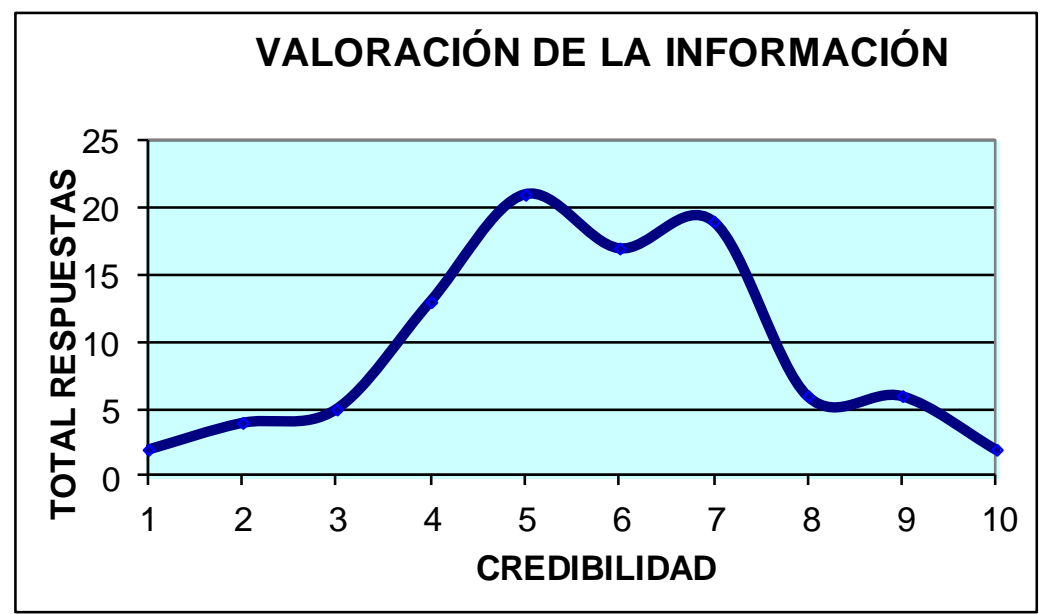

Figura 3: Credibilidad otorgada a la información obtenida de Internet. Media: 5.67.

Datos del INE referentes a porcentajes de búsquedas sobre salud en Internet:

- 2003: $19.57 \%$

- $2007: 41.10 \%$

- $2011: 56.90 \%$ 
Encuesta 2012 realizada en Hospital Viamed Los Manzanos: 64.78\% (tras la eliminación de los cuestionarios recogidos a menores de 16 años, ya que el INE no los refleja en sus datos). (Figura 4).

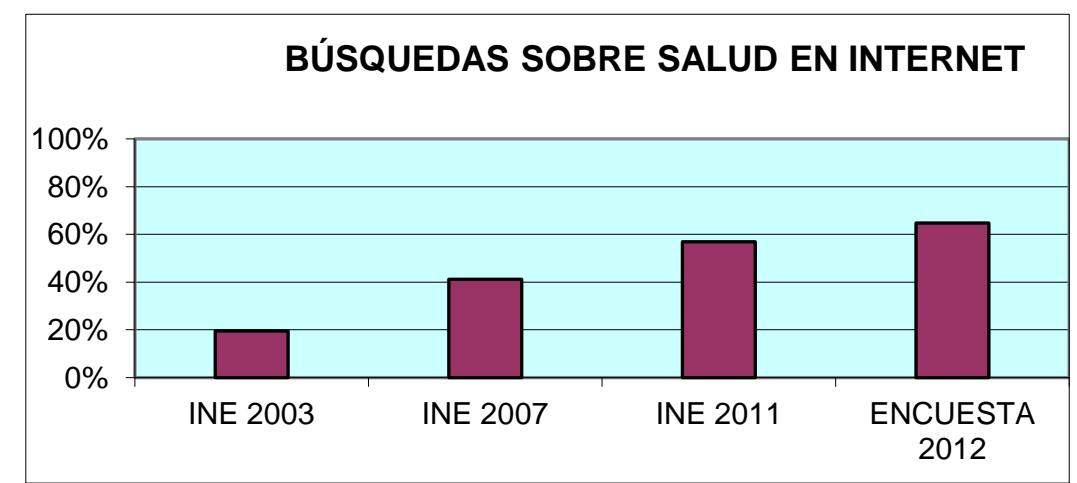

Figura 4: Evolución de las búsquedas sobre salud en Internet. Años 2003, 2007, 2011 y 2012.

\section{DISCUSIÓN}

Nos encontramos con que hasta un $64.78 \%$ de los pacientes hospitalizados y no hospitalizados del Hospital Viamed Los Manzanos consultan Internet para informarse sobre salud. Confrontando los datos de nuestro artículo con otras publicaciones similares sobre el tema, en este caso: Internet como fuente de información en pacientes de atención primaria y su influencia en la relación médico-paciente de Martín Torres et al. ${ }^{(20)}$, observamos datos muy similares en cuanto a porcentajes de búsquedas por parte de los pacientes $(61 \%)$, siendo también muy parejo el porcentaje de búsquedas entre los pacientes menores de 65 años (85.5\% frente a $77.8 \%$ ).

El artículo citado se centra además en evaluar la relación médico-paciente y se alude a la herramienta como "refuerzo de los consejos del médico", lo que podría explicar la falta de diferencias significativas en términos cuantitativos en este estudio, entre los pacientes que se informan en Internet antes de ponerse en contacto con profesionales sanitarios y los que lo hacen después o simplemente podría poner de manifiesto que los pacientes abandonan la consulta con poca información o ésta no es adecuada a su nivel cultural y no son capaces de comprenderla en su totalidad, por lo que precisan informarse también después.

Además, en la comparación de nuestros datos con los ya existentes en el INE para años anteriores, observamos la clara tendencia al alza con el paso de los años de las búsquedas en Internet relacionadas con la salud, tendencia ya observada por Lupiáñez-Villanueva ${ }^{(5)}$, dentro de un concepto más amplio como es el de e-Salud y objetivando una transición de la sociedad industrial a la sociedad Red.

Por otro lado, Jiménez Pernett et al. (11) también evidencian esta evolución ascendente al mostrar aumentos en el uso de Internet en todos los ámbitos y edades, encontrando ellos en su estudio grandes diferencias en cuanto a búsquedas entre mayores de 65 años y ligeramente más jóvenes (55-64 años).

\section{CONCLUSIONES}

Podemos afirmar que la mayoría de los pacientes realizan búsquedas con la intención de informarse sobre salud a través de Internet. Observamos que esta mayoría se 
hace más notoria entre los pacientes más jóvenes, dada la importancia de la edad en el uso de las nuevas tecnologías por razones obvias.

No se advierten diferencias significativas en referencia al porcentaje de acciones de búsqueda de información relativa a la salud entre los pacientes que buscan la información antes de consultar con profesionales sanitarios y los que lo hacen después, lo cual podría dirigirnos hacia una nueva línea de investigación y plantearnos las causas de éste dato. Si Internet es utilizado como complemento a la información dada por parte de los profesionales sanitarios o simplemente si la información proporcionada por parte de los profesionales no es adecuada al nivel cultural de los pacientes y estos consultan Internet para entenderla.

Se aprecia además un marcado crecimiento con el paso de los años de las búsquedas en Internet relacionadas con la salud, con lo que se trata de una herramienta de uso cada vez más difundido y es fundamental estar familiarizado y actualizado en cuanto a la misma.

La credibilidad de 5.67 puntos sobre 10 que se le da a la información obtenida de Internet pone de manifiesto que los encuestados tienen presente que no todo lo que obtienen de la Red es confiable al $100 \%$. No obstante, parece necesario establecer un sistema de control o acreditación de los contenidos referentes a la salud en la Red, dada la gran influencia que tiene sobre los estilos de vida que adopta la población. También consideramos fundamental por parte de los profesionales proporcionar a los pacientes una información correcta, adecuada y continuada, siendo la educación sanitaria una parte imprescindible en la profesión.

\section{Agradecimientos}

Los autores del presente artículo agradecen a la gerencia del Hospital Viamed Los Manzanos su colaboración para la obtención de cuestionarios en sus instalaciones.

Así mismo, quieren felicitar a todo el personal del centro por su buena disposición para la recogida de los mismos.

\section{BIBLIOGRAFÍA}

(1).- Buenaventura R. Las respuestas: Todo lo que usted siempre quiso preguntar sobre Internet. Madrid: Debate; 1999.

(2).- Hafner K., Lyon M. Where Wizards stay up late: The origins of the Internet. United States: Simon \& Schuster; 1998.

(3).- Álvarez Marañón G. Cómo protegernos de los peligros de Internet. Madrid: Consejo Superior de Investigaciones Científicas; 2009.

(4).- Oh H, Rizo C, Enkin M, Jadad A. What is eHealth (3): a systematic review of published definitions. J Med Internet Res. 2005; 7 (1): e1.

(5).- Lupiáñez-Villanueva F. Salud e Internet: más allá de la calidad de la información. Rev Esp Cardiol. 2011; 64 (10): 849-850.

(6).- Lupiáñez-Villanueva F. Salud y sociedad red. Análisis de los usos de Internet relacionados con la salud. Barcelona: Ariel; 2010.

(7).- Instituto Nacional de Estadística (INE) [Internet]. Encuesta sobre equipamiento y uso de Tecnologías de la Información en los hogares 2003, 2007 y 2011. [Consulta el 30/11/2012]. Disponible en: www.ine.es. 
(8).- Del Hoyo Barbolla E., E-health reference framework for personalised information provision to promote sound lifestyles. [Internet]. Tesis Doctoral. T.S.I. Telecomunicación (UPM); 2007.2 Disponible en: http://oa.upm.es/332/1/TESIS_FINAL_EDHB_EDICION_DIGITAL.pdf

(9).- Iverson S.A., Howard K.B., Penney B.K. Impact of the internet use of healthrelated behaviours and the patient-physician relationship: a survey-based study and review. J Am Osteopath Assoc. 2008; 108 (12): 699-711.

(10).- Lalonde M. A new perspective on the health of Canadians. Ottawa: Office of the Canadian Minister of National Health and Welfare; 1974.

(11).- Jiménez Pernett J., García Gutiérrez J.F., Martín Jiménez J.L., Bermúdez Tamayo C. Tendencias en el uso de Internet como fuente de información sobre salud. UOC Papers. [Internet]. 2007. Disponible en: http://www.uoc.edu/uocpapers/4/dt/esp/jimenez.pdf

(12).- Conesa M.C., Aguinaga E. Evaluación de la calidad de las páginas Web con información sanitaria: una revisión bibliográfica. Textos universitaris de biblioteconomia i documentació. 2009; № 23.

(13).- Eysenbach G, Powell J, Kuss O. [et al.]. Empirical studies assessing the quality of health information for consumers on the world wide web. A systematic review. JAMA. 2002; 287(20):2691-2700.

(14).- Berland G. K., Elliott M. N., Morales L. S. [et al.]. Health information on the Internet. Accessibility, quality and readability in English and Spanish. JAMA. 2001; 285(20):2612-2621.

(15).- McCloskey J., Bulechek G.M. Clasificación de Intervenciones de Enfermería (NIC). 5 $5^{\mathrm{a}}$ ed. Madrid: Mosby, 2009.

(16).- Calderero Hernández J.F., Carrasco J.B. Aprendiendo a investigar en educación. 2000. Madrid. RIALP.

(17).- Morales Vallejo P., Urosa Sánz B., Blanco Blanco A. Construcción de escalas de actitudes tipo Likert: una guía práctica. 2003. La Muralla. Madrid.

(18).- Barómetro Sanitario 2011. Ministerio de Sanidad Servicios Sociales e Igualdad. Centro de Investigaciones Sociológicas. [Internet] Disponible en: http://www.msc.es/estadEstudios/estadisticas/docs/BS_2011_total_marginales.pdf

(19).- Kahneman D. Subjective probability: A judgment of representativeness. Cognitive Psychology. 3 (3): 430-454.

(20).- Martín Torres V., Aliaga Valverde J., Sánchez Miró I., Del Castillo Vicente Sáenz M.I., Polentinos-Castro E., Garrido Barral A. Internet como fuente de información para la salud en pacientes de atención primaria y su influencia en la relación médicopaciente. Aten. Primaria. 2012; (12).

ISSN 1695-6141

๑) COPYRIGHT Servicio de Publicaciones - Universidad de Murcia 